UPR-988-T

\title{
Deconstruction of Gauge Symmetry Breaking by Discrete Symmetry and $G^{N}$ Unification
}

\author{
Tianjun $\mathrm{Li}^{1}$ and Tao Liu ${ }^{2}$ \\ Department of Physics and Astronomy \\ University of Pennsylvania, Philadelphia, PA 19104-6396 \\ U. S. A.
}

\begin{abstract}
We deconstruct the non-supersymmetric $S U(5)$ breaking by discrete symmetry on the space-time $M^{4} \times S^{1}$ and $M^{4} \times S^{1} /\left(Z_{2} \times Z_{2}^{\prime}\right)$ in the Higgs mechanism deconstruction scenario. And we explain the subtle point on how to exactly match the continuum results with the latticized results on the quotient space $S^{1} / Z_{2}$ and $S^{1} /\left(Z_{2} \times Z_{2}^{\prime}\right)$. We also propose an effective deconstruction scenario and discuss the gauge symmetry breaking by the discrete symmetry on theory space in this approach. As an application, we suggest the $G^{N}$ unification where $G^{N}$ is broken down to $S U(3) \times S U(2) \times U(1)^{n-3}$ by the bifundamental link fields and the doublet-triplet splitting can be achieved.
\end{abstract}

PACS: 11.25.Mj; 04.65.+e; 11.30.Pb; 12.60.Jv

Keywords: Deconstruction; Gauge Symmetry Breaking; Discrete Symmetry

April 2002

\footnotetext{
${ }^{1}$ Current Address: School of Natural Sciences, Institute for Advanced Study, Einstein Drive, Princeton, NJ 08540. E-mail: tli@sns.ias.edu. Phone Number: (609) 734-8024. Fax Number: (609) 951-4489.

${ }^{2}$ E-mail: liutao@sas.upenn.edu.
} 


\section{Introduction}

Grand Unified Theory (GUT) gives us a simple and elegant understanding of the quantum numbers of quarks and leptons, and the success of gauge coupling unification in the Minimal Supersymmetric Standard Model strongly supports this idea. Although the Grand Unified Theory at high energy scale has been widely accepted, there are some problems in GUT: the grand unified gauge symmetry breaking mechanism, the doublet-triplet splitting problem, and the proton decay problem, etc.

Recently, a new scenario proposed to address above questions in GUT has been discussed extensively $[1,2]$. The key point is that the GUT gauge symmetry exists in 5 or higher dimensions and is broken down to the 4-dimensional $N=1$ supersymmetric Standard Model like gauge symmetry for the zero modes due to the discrete symmetries in the brane neighborhoods or on the extra space manifolds, which become non-trivial constraints on the multiplets and gauge generators in GUT [2]. The attractive models have been constructed explicitly, where the supersymmetric 5-dimensional and 6-dimensional GUT models are broken down to the 4-dimensional $N=1$ supersymmetric $S U(3) \times S U(2) \times U(1)^{n-3}$ model, where $n$ is the rank of GUT group, through the compactification on various orbifolds and manifolds. The GUT gauge symmetry breaking and doublet-triplet splitting problems have been solved neatly by the discrete symmetry projections. Other interesting phenomenology, like $\mu$ problems, gauge coupling unifications, non-supersymmetric GUT, gauge-Higgs unification, proton decay, etc, have also been discussed [1,2].

On the other hand, deconstrution was proposed about one year ago [3]. Deconstruction is interesting because it provides a UV completion of the higher dimensional theories. A lot of phenomenological and formal issues in deconstruction scenarios have been discussed. These include the extensions of the Standard Model, gaugino mediated supersymmetry breaking, low energy unification, GUT breaking, electroweak symmetry breaking, anomaly inflow, the description of little string theories in terms of gauge theory, a single gauge group description of extra dimensions in the large number of color limit, models with noncommutative geometry, warped background geometry, topological objects, Seiberg-Witten curves, and even the deconstruction of time and gravity, etc $[4,5,6,7,8]$. By the way, the arrays of gauge theories, where an infinite number of gauge theories are linked by scalars, were discussed previously [9].

In this paper, we would like to discuss the deconstruction of gauge symmetry breaking by the discrete symmetry on extra space manifold. If we know how to deconstruct those higher dimensional theories, we can have a lot of good features in deonstruction scenarios, for example, the gauge symmetry breaking, the doublet-triplet splitting, suppressing the proton decay, the gauge-Higgs unification (In the deconstruction language, bifundamental fields and $S U(2)_{L}$ Higgs unification.). For simplicity, we do not consider supersymmetry. First, we consider the Higgs mechanism deconstruction scenario in which the gauge bosons obtain the masses via the VEVs of the bifundamental link Higgs fields. We shall deconstruct the non-supersymmetric $S U(5)$ breaking by the discrete symmetry on the space-time $M^{4} \times S^{1}$ and $M^{4} \times S^{1} /\left(Z_{2} \times Z_{2}^{\prime}\right)$ where $M^{4}$ is the 4-dimensional Minkowski space-time. We also explain the subtle 
point on how to exactly match the continuum results with the latticized results on the quotient space $S^{1} / Z_{2}$ and $S^{1} /\left(Z_{2} \times Z_{2}^{\prime}\right)$. In addition, it seems to us that the Higgs mechanism deconstructions of the gauge symmetry breaking by discrete symmetry on the space-time $M^{4} \times S^{1}$ and $M^{4} \times S^{1} /\left(Z_{2} \times Z_{2}^{\prime}\right)$ might not be the real deconstructions. The key point is that, the bifundamental field $U_{i}$, which is the Schwinger line integral along the fifth dimension, should be considered as the gauge field $A_{5}$. However, the mass spectrum and the 5-dimensional wave functions for the KK modes of $A_{5}$ can not match those for $U_{i}$ in the Higgs mechanism deconstruction scenario by counting the massless modes ${ }^{3}$. Therefore, we propose the effective deconstruction scenario where we add the mass term for each field by hand which comes from the latticization of the kinetic term of that field along the fifth dimension. In the effective deconstruction scenario, similar to the gauge symmetry breaking by the discrete symmetry on extra space manifold, we can define the discrete symmetry on theory space and discuss the gauge symmetry breaking. Moreover, we show that the continuum results match the effective deconstruction results exactly. As an application, we discuss the $G^{N}$ unification where $G^{N}$ is broken down to $S U(3) \times S U(2) \times U(1)^{n-3}$ ( $n$ is the rank of group $G)$ by the bifundamental link fields and the doublet-triplet splitting can be achieved. With the general $G^{N}$ unification, we wish we can solve the tough problems in the traditional 4-dimensional GUT models.

Let us explain our terminology. The exactly match between the $n-t h \mathrm{KK}$ mode of a bulk field in the continuum case and the corresponding state in the deconstruction case means that the $n-t h$ mass eigenvalue and eigenvector of the field in the deconstruction scenario are the same as the mass and 5-dimensional wave function of the $n-t h \mathrm{KK}$ mode of the corresponding bulk field when $N>>n$.

\section{Higgs Mechanism Deconstruction of $S U(5)$ Break- ing on $M^{4} \times S^{1}$ by Wilson Line}

In this section, we would like to deconstruct the non-supersymmetric $S U(5)$ breaking on the space-time $M^{4} \times S^{1}$ by Wilson line in the Higgs mechanism deconstruction scenario. And we want to point out that one can discuss any other GUT groups similarly because the fundamental group of $S^{1}$ is $Z, i$. e., $\pi_{1}\left(S^{1}\right)=Z[2]$.

\section{1 $S U(5)$ Breaking on $M^{4} \times S^{1}$ by Wilson Line}

Let us consider the 5-dimensional space-time which can be factorized into a product of the ordinary 4-dimensional Minkowski space-time $M^{4}$ and the circle $S^{1}$. The

\footnotetext{
${ }^{3}$ One might consider the axial gauge $A_{5}=0$ in the 5-dimensional theory. However, only part of the scalars from $U_{i}$ fields are eaten by the massive gauge bosons after the gauge symmetry breaking, and most of the physical scalars from $U_{i}$ fields can obtain masses via Higgs mechanism. Therefore, the 5-dimensional gauge field $A_{5}$ in the axial gauge $\left(A_{5}=0\right)$ can not match the bifundamental fields $U_{i}$ in the Higgs mechanism deconstruction scenario, too.
} 
corresponding coordinates are $x^{\mu},(\mu=0,1,2,3), y \equiv x^{5}$, and the radius for the fifth dimension is $R$. The gauge fields are denoted as $A_{M}\left(x^{\mu}, y\right)$ where $M=0,1,2,3,5$. Because $Z_{2} \subset \pi_{1}\left(S^{1}\right)$, we can define the $Z_{2}$ parity operator $P$ for a generic bulk multiplet $\Phi\left(x^{\mu}, y\right)$

$$
\Phi\left(x^{\mu}, y\right) \rightarrow \Phi\left(x^{\mu}, y+2 \pi R\right)=\eta_{\Phi} P^{l_{\Phi}} \Phi\left(x^{\mu}, y\right)\left(P^{-1}\right)^{m_{\Phi}}
$$

where $\eta_{\Phi}= \pm 1$ and $P^{2}=1$. By the way, if the gauge group $G$ is $S U(5)$, for a 5 -plet $\Phi$ in the fundamental representation, $l_{\Phi}=1$ and $m_{\Phi}=0$, and for a 24-plet $\Phi$ in the adjoint representation, $l_{\Phi}=1$ and $m_{\Phi}=1$.

Denoting the field $\phi$ with parity $P= \pm$ by $\phi_{ \pm}$, we obtain the KK mode expansions

$$
\begin{gathered}
\phi_{+}\left(x^{\mu}, y\right)=\sum_{n=-\infty}^{+\infty} \phi_{+}^{n}\left(x^{\mu}\right) e^{i \frac{n y}{R}}, \\
\phi_{-}\left(x^{\mu}, y\right)=\sum_{n=-\infty}^{+\infty} \phi_{-}^{n}\left(x^{\mu}\right) e^{i \frac{(n+1 / 2) y}{R}} .
\end{gathered}
$$

Now, let us discuss the $S U(5)$ breaking. Under parity $P$, the gauge fields $A_{M}$ transform as

$$
A_{M}\left(x^{\mu}, y+2 \pi R\right)=P A_{M}\left(x^{\mu}, y\right) P^{-1}
$$

And we choose the following matrix representation for parity operator $P$, which is expressed in the adjoint representation of $S U(5)$

$$
P=\operatorname{diag}(-1,-1,-1,+1,+1) .
$$

So, upon the $P$ parity, the gauge generators $T^{A}$ where $\mathrm{A}=1,2, \ldots, 24$ for $S U(5)$ are separated into two sets: $T^{a}$ are the gauge generators for the Standard Model gauge group, and $T^{\hat{a}}$ are the other broken gauge generators

$$
P T^{a} P^{-1}=T^{a}, P T^{\hat{a}} P^{-1}=-T^{\hat{a}} .
$$

And the masses for $A_{M}^{a}$ and $A_{M}^{\hat{a}}$ are $n / R$ and $(n+1 / 2) / R$, respectively. In addition, if we add a pair of Higgs 5-plets $H_{u}$ and $H_{d}$ in the bulk, then for each 5-plet, the doublet mass is $n / R$ and the triplet mass is $(n+1 / 2) / R$ if $\eta_{H_{u}}=\eta_{H_{d}}=+1$. In short, for the zero modes, the gauge group $S U(5)$ is broken down to $S U(3) \times S U(2) \times U(1)$, and we can solve the doublet-triplet splitting problem. The parities and masses of the fields in the $S U(5)$ gauge and Higgs multiplets are given in Table 1. 
Table 1: Parity assignment and masses of the fields in the $S U(5)$ gauge and Higgs multiplets for the model with $S U(5)$ breaking by Wilson line. The index $a$ labels the unbroken $S U(3) \times S U(2) \times U(1)$ gauge generators, while $\hat{a}$ labels the other broken SU(5) gauge generators. The indices $D$ and $T$ are for doublet and triplet, respectively.

\begin{tabular}{|c|c|c|}
\hline$P$ & field & mass $(n=0, \pm 1, \pm 2, \ldots)$ \\
\hline+ & $A_{\mu}^{a}, A_{5}^{a}, H_{u}^{D}, H_{d}^{D}$ & $n / R$ \\
\hline- & $A_{\mu}^{\hat{a}}, A_{5}^{\hat{a}} H_{u}^{T}, H_{d}^{T}$ & $(n+1 / 2) / R$ \\
\hline
\end{tabular}

\subsection{Higgs Mechanism Deconstruction}

We consider the $S U(5)^{N+1}$ gauge theory with bifundamental fields $U_{i}$ as follows

\begin{tabular}{c|cccccc} 
& $S U(5)_{0}$ & $S U(5)_{1}$ & $S U(5)_{2}$ & $\cdots$ & $S U(5)_{N-1}$ & $S U(5)_{N}$ \\
\hline$U_{0}$ & $\square$ & $\square$ & 1 & $\cdots$ & 1 & 1 \\
$U_{1}$ & 1 & $\square$ & $\square$ & $\cdots$ & 1 & 1 \\
$\vdots$ & $\vdots$ & $\vdots$ & $\ddots$ & $\vdots$ & $\vdots$ & \\
$U_{N-1}$ & 1 & 1 & 1 & $\cdots$ & $\square$ & $\square$ \\
$U_{N}$ & $\square$ & 1 & 1 & $\cdots$ & 1 & $\square$
\end{tabular}

And the effective action is

$$
S=\int d^{4} x \sum_{i=0}^{N}\left(-\frac{1}{4 g^{2}} \operatorname{Tr} F_{i}^{2}+\operatorname{Tr}\left[\left(D_{\mu} U_{i}\right)^{\dagger} D^{\mu} U_{i}\right]+\ldots\right),
$$

where the covariant derivative is $D^{\mu} U_{i} \equiv \partial_{\mu} U_{i}-i A_{\mu}^{i} U_{i}+i U_{i} A_{\mu}^{i+1}$ and the dots represent the higher dimensional operators that are irrelevant at low energies.

The bifundamental fields $U_{i}$ obtain the vacuum expectation values (VEV) either from a suitable renormalizable potential or from some strong interactions. In order to obtain the deconstruction of the Wilson line gauge symmetry breaking, we choose the following VEVs for $U_{i}$

$$
\begin{aligned}
<U_{i}> & =\operatorname{diag}(v / \sqrt{2}, v / \sqrt{2}, v / \sqrt{2}, v / \sqrt{2}, v / \sqrt{2}), \text { for } i=0,1,2, \ldots, N-1, \\
& <U_{N}>=\operatorname{diag}(-v / \sqrt{2},-v / \sqrt{2},-v / \sqrt{2}, v / \sqrt{2}, v / \sqrt{2}) .
\end{aligned}
$$


We would like to explain the VEV for $U_{N}$, which is different from the VEV for $U_{i}$ where $i=0,1,2, \ldots, N-1$. In general, we can take that the VEV for $U_{N}$ is the same as that for $U_{i}$. And the masses for gauge bosons are given by the mass terms $\frac{1}{2} \sum_{i=0}^{N} g^{2} v^{2}\left(A_{\mu}^{\beta(i+1)}-A_{\mu}^{\beta i}\right)^{2}$, where $A_{\mu}^{\beta(N+1)}=A_{\mu}^{\beta 0}$ for $\beta=a$ and $A_{\mu}^{\beta(N+1)}=-A_{\mu}^{\beta 0}$ for $\beta=\hat{a}$ due to the Wilson line gauge symmetry breaking (See Table 1). However, in the Higgs mechanism deconstruction scenario, we take $A_{\mu}^{\beta(N+1)}=A_{\mu}^{\beta 0}$ for $\beta=a$ and $\beta=\hat{a}$, so, the last term in above mass terms is $\frac{1}{2} g^{2} v^{2}\left(A_{\mu}^{\beta 0}-A_{\mu}^{\beta N}\right)^{2}$ for $\beta=a$, and $\frac{1}{2} g^{2} v^{2}\left(A_{\mu}^{\beta 0}+A_{\mu}^{\beta N}\right)^{2}$ for $\beta=\hat{a}$. This correct mass term can be obtained by choosing the suitable VEV for $U_{N}$, which is the Eq. (10) in our model. By the way, we emphasize that the VEV for $U_{N}$ in Eq. (10) is similar to the matrix representation for parity operator $P$ in Eq. (5), which breaks the $S U(5)$ gauge symmerty.

Thus, the $(N+1) \times(N+1)$ mass matrix for the Standard Model gauge boson or the column vector $\left(A_{\mu}^{a 0}, A_{\mu}^{a 1}, A_{\mu}^{a 2}, \ldots, A_{\mu}^{a N}\right)$ is

$$
M_{S M}^{2}=g^{2} v^{2}\left(\begin{array}{ccccc}
2 & -1 & 0 & \cdots & -1 \\
-1 & 2 & -1 & \cdots & 0 \\
0 & -1 & 2 & \cdots & 0 \\
& & & \cdots & \\
-1 & 0 & \cdots & -1 & 2
\end{array}\right) \text {. }
$$

The mass spectrum or eigenvalue is

$$
M_{n}^{2}=4 g^{2} v^{2} \sin ^{2}\left(\frac{n \pi}{N+1}\right),
$$

where $-N / 2 \leq n \leq N / 2$ or $n=0,1,2, \ldots, N$. And the corresponding $n-t h$ eigenvector is

$$
\alpha_{n}=\left(\alpha_{n}^{0}, \alpha_{n}^{1}, \ldots, \alpha_{n}^{N}\right),
$$

where

$$
\alpha_{n}^{j}=\frac{1}{\sqrt{N+1}} \exp \left(i \frac{2 j n \pi}{N+1}\right), j=0,1, \ldots, N .
$$

Noticing that $R=(N+1) /(g v)$, we obtain that the $n$-th eigenvector matches the 5 dimensional wave function $\left(e^{i \frac{n u}{R}}\right)$ of the $n-t h \mathrm{KK}$ mode for $A_{\mu}^{a}$ in the last subsection, and for $n<<N$, the $n-t h$ mass (eigenvalue) matches the mass of the $n-t h \mathrm{KK}$ mode for $A_{\mu}^{a}$. So, they exactly match.

And the mass matrix for the non-Standard Model gauge boson or the column 
$\operatorname{vector}\left(A_{\mu}^{\hat{a} 0}, A_{\mu}^{\hat{a} 1}, A_{\mu}^{\hat{a} 2}, \ldots, A_{\mu}^{\hat{a} N}\right)$ is

$$
M_{N S M}^{2}=g^{2} v^{2}\left(\begin{array}{ccccc}
2 & -1 & 0 & \cdots & +1 \\
-1 & 2 & -1 & \cdots & 0 \\
0 & -1 & 2 & \cdots & 0 \\
& & & \cdots & \\
+1 & 0 & \cdots & -1 & 2
\end{array}\right) \text {. }
$$

The mass spectrum or eigenvalue is

$$
M_{n}^{2}=4 g^{2} v^{2} \sin ^{2}\left(\frac{(n+1 / 2) \pi}{N+1}\right)
$$

where $-N / 2 \leq n \leq N / 2$ or $n=0,1,2, \ldots, N$. And the corresponding $n-t h$ eigenvector is

$$
\alpha_{n}=\left(\alpha_{n}^{0}, \alpha_{n}^{1}, \ldots, \alpha_{n}^{N}\right)
$$

where

$$
\alpha_{n}^{j}=\frac{1}{\sqrt{N+1}} \exp \left(i \frac{j(2 n+1) \pi}{N+1}\right), j=0,1, \ldots, N
$$

Noticing that $R=(N+1) /(g v)$, we obtain that the $n-t h$ eigenvector matches the 5 -dimensional wave function $\left(e^{i \frac{(n+1 / 2) y}{R}}\right)$ of the $n-t h \mathrm{KK}$ mode for $A_{\mu}^{\hat{a}}$ in the last subsection, and for $n<<N$, the $n-t h$ mass (eigenvalue) matches the mass of the $n-t h \mathrm{KK}$ mode for $A_{\mu}^{\hat{a}}$. Thus, they exactly match, and we want to emphasize that there is no massless mode for $A_{\mu}^{\hat{a} i}$.

In addition, we can discuss the deconstruction of Higgs fields $H_{u}$ and $H_{d}$. We assume that under the $i-t h$ gauge group $S U(5)_{i}$, there is a pair of Higgs 5 -plets $H_{u}^{i}$ and $H_{d}^{i}$ where $i=0,1,2, \ldots, N$. And we consider the following potential

$$
\begin{aligned}
V= & 2 g^{2} \sum_{i=0}^{N}\left(\left|U_{i}\left(H_{u}^{i}\right)^{\dagger}\right|^{2}+\left|H_{u}^{i+1} U_{i}\right|^{2}+\left|U_{i} H_{d}^{i}\right|^{2}+\left|\left(H_{d}^{i+1}\right)^{\dagger} U_{i}\right|^{2}\right) \\
& -\sqrt{2} g^{2} v \sum_{i=0}^{N}\left(H_{u}^{i+1} U_{i}\left(H_{u}^{i}\right)^{\dagger}+\left(H_{d}^{i+1}\right)^{\dagger} U_{i} H_{d}^{i}+\text { H.C. }\right)
\end{aligned}
$$

where for simplicity, we define $H_{u}^{N+1} \equiv H_{u}^{0}$ and $H_{d}^{N+1} \equiv H_{d}^{0}$. The above potential for a pair of Higgs 5-plets $H_{u}^{i}$ and $H_{d}^{i}$ comes from the deconstruction of $\left(D_{5} H_{u}\right)^{\dagger} D_{5} H_{u}$ and $\left(D_{5} H_{d}\right)^{\dagger} D_{5} H_{d}$ in 5-dimensional theory, and the coefficients are determined by the normalization which is compatible with that of gauge fields. In short, we obtain that the mass matrix for the doublet $H_{u}^{i D}$ or $H_{d}^{i D}$ is the same as that for the Standard Model gauge boson in Eq. (11), and the mass matrix for the triplet $H_{u}^{i T}$ or $H_{d}^{i T}$ is the 
same as that for the non-Standard Model gauge boson in Eq. (15). Therefore, similar to the discussions for $A_{\mu}^{a}$ and $A_{\mu}^{\hat{a}}$, the deconstruction results match the continuum results in Table 1. And then, we solve the doublet-triplet splitting problem.

Furthermore, by counting the number of massless modes, we can prove that the bifundamental fields $U_{i}$ can not match the gauge fields $A_{5}$ because $U_{i}$ s are Higgs fields. The correct deconstruction of the $A_{5}$ fields should have 12 massless modes, but, the $U_{i}$ s field will have at least $12(2 N+1)$ massless modes that are the Goldstone bosons and give masses to the longitudinal components of massive gauge bosons.

In short, the gauge group is broken down to $S U(3) \times S U(2) \times U(1)$ for the zero modes, and the deconstruction results match the continuum results except for $A_{5}$ and $U_{i}$.

\section{Higgs Mechanism Deconstruction of $S U(5)$ Break- ing on $M^{4} \times S^{1} /\left(Z_{2} \times Z_{2}^{\prime}\right)$}

In this section, we would like to discuss the non-supersymmetric $S U(5)$ breaking on the space-time $M^{4} \times S^{1} /\left(Z_{2} \times Z_{2}^{\prime}\right)$. By the way, the $S U(5)$ breaking on the space-time $M^{4} \times S^{1} / Z_{2}$ can be discussed similarly.

\section{1 $S U(5)$ Breaking on $M^{4} \times S^{1} /\left(Z_{2} \times Z_{2}^{\prime}\right)$}

Our convention is similar to that in the subsection 2.1. The orbifold $S^{1} /\left(Z_{2} \times Z_{2}^{\prime}\right)$ is obtained by $S^{1}$ moduloing the following equivalent classes:

$$
y \sim-y, y^{\prime} \sim-y^{\prime},
$$

where $y^{\prime}$ is defined as $y^{\prime} \equiv y-\pi R / 2$.

For a generic bulk multiplet $\Phi\left(x^{\mu}, y\right)$ which fills a representation of the gauge group $G$, we can define two parity operators $P$ and $P^{\prime}$ for the $Z_{2}$ and $Z_{2}^{\prime}$ symmetries, respectively

$$
\begin{gathered}
\Phi\left(x^{\mu}, y\right) \rightarrow \Phi\left(x^{\mu},-y\right)=\eta_{\Phi} P^{l_{\Phi}} \Phi\left(x^{\mu}, y\right)\left(P^{-1}\right)^{m_{\Phi}}, \\
\Phi\left(x^{\mu}, y^{\prime}\right) \rightarrow \Phi\left(x^{\mu},-y^{\prime}\right)=\eta_{\Phi}^{\prime}\left(P^{\prime}\right)^{l_{\Phi}} \Phi\left(x^{\mu}, y^{\prime}\right)\left(P^{\prime-1}\right)^{m_{\Phi}},
\end{gathered}
$$

where $\eta_{\Phi}= \pm 1$ and $\eta_{\Phi}^{\prime}= \pm 1$.

Denoting the field $\phi$ with $\left(P, P^{\prime}\right)=( \pm, \pm)$ by $\phi_{ \pm \pm}$, we obtain the KK mode expansions

$$
\begin{gathered}
\phi_{++}\left(x^{\mu}, y\right)=\sum_{n=0}^{\infty} \frac{1}{\sqrt{2^{\delta_{n, 0}} \pi R}} \phi_{++}^{(2 n)}\left(x^{\mu}\right) \cos \frac{2 n y}{R}, \\
\phi_{+-}\left(x^{\mu}, y\right)=\sum_{n=0}^{\infty} \frac{1}{\sqrt{\pi R}} \phi_{+-}^{(2 n+1)}\left(x^{\mu}\right) \cos \frac{(2 n+1) y}{R},
\end{gathered}
$$




$$
\begin{aligned}
& \phi_{-+}\left(x^{\mu}, y\right)=\sum_{n=0}^{\infty} \frac{1}{\sqrt{\pi R}} \phi_{-+}^{(2 n+1)}\left(x^{\mu}\right) \sin \frac{(2 n+1) y}{R}, \\
& \phi_{--}\left(x^{\mu}, y\right)=\sum_{n=0}^{\infty} \frac{1}{\sqrt{\pi R}} \phi_{--}^{(2 n+2)}\left(x^{\mu}\right) \sin \frac{(2 n+2) y}{R} .
\end{aligned}
$$

The 4-dimensional fields $\phi_{++}^{(2 n)}, \phi_{+-}^{(2 n+1)}, \phi_{-+}^{(2 n+1)}$ and $\phi_{--}^{(2 n+2)}$ acquire masses $2 n / R,(2 n+$ $1) / R,(2 n+1) / R$ and $(2 n+2) / R$ upon the compactification. Zero modes are contained only in $\phi_{++}$fields, thus, the matter content of massless sector is smaller than that of the full 5-dimensional multiplet. Moreover, only $\phi_{++}$and $\phi_{+-}$fields have non-zero values at $y=0$, and only $\phi_{++}$and $\phi_{-+}$fields have non-zero values at $y=\pi R / 2$.

Under parity $P$, the gauge fields $A_{M}$ transform as

$$
\begin{aligned}
& A_{\mu}\left(x^{\mu},-y\right)=P A_{\mu}\left(x^{\mu}, y\right) P^{-1} \\
& A_{5}\left(x^{\mu},-y\right)=-P A_{5}\left(x^{\mu}, y\right) P^{-1} .
\end{aligned}
$$

And under parity $P^{\prime}$, the gauge field transformations are similar to those under $P$.

We choose the following matrix representations for parity operators $P$ and $P^{\prime}$ that are expressed in the adjoint representation of $\mathrm{SU}(5)$

$$
P=\operatorname{diag}(+1,+1,+1,+1,+1), P^{\prime}=\operatorname{diag}(-1,-1,-1,+1,+1) .
$$

So, under $P^{\prime}$ parity, the $S U(5)$ gauge generators $T^{A}$ where $\mathrm{A}=1,2, \ldots, 24$ for $\mathrm{SU}(5)$ are separated into two sets: $T^{a}$ are the gauge generators for the Standard Model gauge group, and $T^{\hat{a}}$ are the other broken gauge generators

$$
\begin{gathered}
P T^{a} P^{-1}=T^{a}, P T^{\hat{a}} P^{-1}=T^{\hat{a}}, \\
P^{\prime} T^{a}\left(P^{\prime}\right)^{-1}=T^{a}, P^{\prime} T^{\hat{a}}\left(P^{\prime}\right)^{-1}=-T^{\hat{a}} .
\end{gathered}
$$

Therefore, the masses for gauge fields $A_{\mu}^{a}, A_{\mu}^{\hat{a}}, A_{5}^{a}$ and $A_{5}^{\hat{a}}$ are $2 n / R,(2 n+1) / R$, $(2 n+2) / R$ and $(2 n+1) / R$, respectively. For the zero modes, the gauge group is $S U(3) \times S U(2) \times U(1)$. Including the KK modes, the gauge groups at $y=0$ and $y=\pi R / 2$ are $S U(5)$ and $S U(3) \times S U(2) \times U(1)$, respectively.

Moreover, assuming that there exists a pair of Higgs 5-plets $H_{u}$ and $H_{d}$ in the bulk and $\eta_{H_{u}}=\eta_{H_{d}}=+1$, we obtain that for each 5 -plet, the doublet mass is $2 n / R$, and the triplet mass is $(2 n+1) / R$. So, we solve the doublet-triplet splitting problem. The parities and masses of the fields in the $S U(5)$ gauge and Higgs multiplets are given in Table 2. 
Table 2: Parity assignment and masses of the fields $(n \geq 0)$ in the $S U(5)$ gauge and Higgs multiplets for the model with $S U(5)$ breaking on $M^{4} \times S^{1} /\left(Z_{2} \times Z_{2}^{\prime}\right)$.

\begin{tabular}{|c|c|c|}
\hline$\left(P, P^{\prime}\right)$ & field & mass $(n=0,1,2, \ldots)$ \\
\hline$(+,+)$ & $A_{\mu}^{a}, H_{u}^{D}, H_{d}^{D}$ & $2 n / R$ \\
\hline$(+,-)$ & $A_{\mu}^{\hat{a}}, H_{u}^{T}, H_{d}^{T}$ & $(2 n+1) / R$ \\
\hline$(-,+)$ & $A_{5}^{\hat{a}}$ & $(2 n+1) / R$ \\
\hline$(-,-)$ & $A_{5}^{a}$ & $(2 n+2) / R$ \\
\hline
\end{tabular}

\subsection{Higgs Mechanism Deconstruction}

We consider the $S U(5)^{N} \times(S U(3) \times S U(2) \times U(1))$ gauge theory with bifundamental fields $U_{i}(i=0,1, \ldots, N-1), U_{c}$ and $U_{w}$ as follows

\begin{tabular}{c|cccccccc} 
& $S U(5)_{0}$ & $S U(5)_{1}$ & $S U(5)_{2}$ & $\cdots$ & $S U(5)_{N-1}$ & $S U(3)$ & $S U(2)$ & $U(1)_{Y}$ \\
\hline$U_{0}$ & $\square$ & $\square$ & 1 & $\cdots$ & 1 & 1 & 1 & 0 \\
$U_{1}$ & 1 & $\square$ & $\square$ & $\cdots$ & 1 & 1 & 1 & 0 \\
$U_{2}$ & 1 & 1 & $\square$ & $\cdots$ & 1 & 1 & 1 & 0 \\
$\vdots$ & $\vdots$ & $\vdots$ & $\vdots$ & $\ddots$ & $\vdots$ & $\vdots$ & $\vdots$ & $\vdots$ \\
$U_{c}$ & 1 & 1 & 1 & $\ldots$ & $\square$ & $\square$ & 1 & $\frac{1}{3}$ \\
$U_{w}$ & 1 & 1 & 1 & $\cdots$ & $\square$ & 1 & $\square$ & $-\frac{1}{2}$
\end{tabular}

This kind of models has been discussed recently in Ref $[5,7]$.

The effective action is

$$
\begin{aligned}
S= & \int d^{4} x \sum_{i=0}^{N-2}\left(-\frac{1}{4 g^{2}} \operatorname{Tr} F_{i}^{2}+\operatorname{Tr}\left[\left(D_{\mu} U_{i}\right)^{\dagger} D^{\mu} U_{i}\right]+\ldots\right) \\
& -\frac{1}{4 g^{2}} \operatorname{Tr} F_{c}^{2}+\operatorname{Tr}\left[\left(D_{\mu} U_{c}\right)^{\dagger} D^{\mu} U_{c}\right]+\ldots \\
& -\frac{1}{4 g^{2}} \operatorname{Tr} F_{w}^{2}+\operatorname{Tr}\left[\left(D_{\mu} U_{w}\right)^{\dagger} D^{\mu} U_{w}\right]+\ldots .
\end{aligned}
$$

Because we consider the Higgs mechanism deconstruction of $S U(5)$ breaking on the space-time $M^{4} \times S^{1} /\left(Z_{2} \times Z_{2}^{\prime}\right)$, at GUT scale, we should take the same gauge couplings for all the gauge groups $S U(5)^{N} \times(S U(3) \times S U(2) \times U(1))$.

And we choose the following $\mathrm{VEVs}$ for $U_{i}, U_{c}$ and $U_{w}$

$$
<U_{i}>=\operatorname{diag}(v / \sqrt{2}, v / \sqrt{2}, v / \sqrt{2}, v / \sqrt{2}, v / \sqrt{2}) \text {, for } i=0,1,2, \ldots, N-1 \text {, }
$$




$$
<U_{c}>=\operatorname{diag}(v / \sqrt{2}, v / \sqrt{2}, v / \sqrt{2}), \quad<U_{w}>=\operatorname{diag}(v / \sqrt{2}, v / \sqrt{2}) .
$$

The $(N+1) \times(N+1)$ mass matrix for the Standard Model gauge boson is

$$
M_{S M}^{2}=g^{2} v^{2}\left(\begin{array}{ccccccc}
1 & -1 & 0 & \cdots & 0 & 0 & 0 \\
-1 & 2 & -1 & \cdots & 0 & 0 & 0 \\
0 & -1 & 2 & \cdots & 0 & 0 & 0 \\
& & & \ddots & & & \\
0 & 0 & 0 & \cdots & -1 & 2 & -1 \\
0 & 0 & 0 & \cdots & 0 & -1 & 1
\end{array}\right) \text {. }
$$

However, the mass spectrum and eigenvector of above mass matrix do not exactly match those of $A_{\mu}^{a}$ in the last subsection because of the fixed point. The subtle point is similar to that in the brane models on $M^{4} \times S^{1} / Z_{2}$ or $M^{4} \times R^{1} / Z_{2}$ where the brane tension at fixed point on the quotient space is half of that on the covering space [10]. And on the covering space $S^{1}$, the mass matrix for the Standard Model gauge boson is similar to that in Eq. (11), then the continuum results and the deconstruction results do exactly match. In short, the $n-t h$ column eigenvector $\alpha_{n}$ and eigenvalue $M_{n}^{2}$ should satisfy the following equation

$$
\left(M_{S M}^{2}\right)_{i j} \alpha_{n}^{j}=\left[1-\frac{1}{2}\left(\delta_{0 i}+\delta_{N i}\right)\right] M_{n}^{2} \alpha_{n}^{i}
$$

The mass spectrum or eigenvalue is

$$
M_{n}^{2}=4 g^{2} v^{2} \sin ^{2}\left(\frac{n \pi}{2 N}\right),
$$

where $n=0,1,2, \ldots, N$. And the corresponding $n-t h$ eigenvector is

$$
\alpha_{n}=\left(\alpha_{n}^{0}, \alpha_{n}^{1}, \ldots, \alpha_{n}^{N}\right),
$$

where

$$
\alpha_{n}^{j}=\frac{\sqrt{2}}{\sqrt{N+1}} \cos \left(\frac{2 j n \pi}{2 N}\right), j=0,1, \ldots, N .
$$

Noticing that $R=2 N /(g v)$, we obtain that the $n-t h$ eigenvector matches the 5dimensional wave function $(\cos (2 n y / R))$ of the $n-t h \mathrm{KK}$ mode for $A_{\mu}^{a}$ in the last subsection $\left(\phi_{++}\right)$, and for $n<<N$, the $n-t h$ mass (eigenvalue) matches the mass of the $n-t h \mathrm{KK}$ mode for $A_{\mu}^{a}$ in the last subsection. 
The $N \times N$ mass matrix for the non-Standard Model gauge boson is

$$
M_{N S M}^{2}=g^{2} v^{2}\left(\begin{array}{ccccccc}
1 & -1 & 0 & \cdots & 0 & 0 & 0 \\
-1 & 2 & -1 & \cdots & 0 & 0 & 0 \\
0 & -1 & 2 & \cdots & 0 & 0 & 0 \\
& & & \ddots & & & \\
0 & 0 & 0 & \cdots & -1 & 2 & -1 \\
0 & 0 & 0 & \cdots & 0 & -1 & 2
\end{array}\right) \text {. }
$$

Similarly, the mass spectrum and eigenvector of above matrix do not match those of $A_{\mu}^{\hat{a}}$ in the last subsection due to the fixed point. On the covering space $S^{1}$, the mass matrix is reducible and the irreducible mass matrix is similar to that in Eq. (50). Thus, the continuum results and the deconstruction results do exactly match on the covering space $S^{1}$. In short, the $n-t h$ eigenvector $\alpha_{n}$ and eigenvalue $M_{n}^{2}$ should satisfy the following equation

$$
\left(M_{N S M}^{2}\right)_{i j} \alpha_{n}^{j}=\left[1-\frac{1}{2} \delta_{0 i}\right] M_{n}^{2} \alpha_{n}^{i},
$$

where $j=0,1,2, \ldots, N-1$. The mass spectrum or eigenvalue is

$$
M_{n}^{2}=4 g^{2} v^{2} \sin ^{2}\left(\frac{(2 n+1) \pi}{4 N}\right),
$$

where $n=0,1,2, \ldots, N-1$. And the corresponding $n-t h$ eigenvector is

$$
\alpha_{n}=\left(\alpha_{n}^{0}, \alpha_{n}^{1}, \ldots, \alpha_{n}^{N-1}\right),
$$

where

$$
\alpha_{n}^{j}=\frac{\sqrt{2}}{\sqrt{N}} \cos \left(\frac{j(2 n+1) \pi}{2 N}\right), j=0,1, \ldots, N-1 .
$$

Noticing that $R=2 N /(g v)$, we obtain that the $n$ - th eigenvector matches the 5dimensional wave function $(\cos ((2 n+1) y / R))$ of the $n-t h$ KK mode for $A_{\mu}^{\hat{a}}$ in the last subsection $\left(\phi_{+-}\right)$, and for $n<<N$, the $n-t h$ mass (eigenvalue) matches the mass of the $n$ - th KK mode for $A_{\mu}^{\hat{a}}$ in the last subsection.

In addition, we would like to discuss the deconstruction of 5-dimensional fields $\phi_{-+}$and $\phi_{--}$because we will have this kind of field expansions when we discuss the effective deconstruction scenario in the next section. So, let us give the mass matrix, eigenvalues and eigenvectors here. 
The $N \times N$ mass matrix for the deconstruction of 5-dimensional field $\phi_{-+}$is

$$
M_{-+}^{2}=g^{2} v^{2}\left(\begin{array}{ccccccc}
2 & -1 & 0 & \cdots & 0 & 0 & 0 \\
-1 & 2 & -1 & \cdots & 0 & 0 & 0 \\
0 & -1 & 2 & \cdots & 0 & 0 & 0 \\
& & & \ddots & & & \\
0 & 0 & 0 & \cdots & -1 & 2 & -1 \\
0 & 0 & 0 & \cdots & 0 & -1 & 1
\end{array}\right) .
$$

The mass spectrum or eigenvalue is

$$
M_{n}^{2}=4 g^{2} v^{2} \sin ^{2}\left(\frac{(2 n+1) \pi}{4 N}\right),
$$

where $n=0,1,2, \ldots, N-1$. And the corresponding $n-t h$ eigenvector is

$$
\alpha_{n}=\left(\alpha_{n}^{1}, \alpha_{n}^{2}, \ldots, \alpha_{n}^{N}\right),
$$

where

$$
\alpha_{n}^{j}=\frac{\sqrt{2}}{\sqrt{N}} \sin \left(\frac{j(2 n+1) \pi}{2 N}\right), j=1,2, \ldots, N .
$$

The $(N-1) \times(N-1)$ mass matrix for the deconstruction of 5-dimensional field $\phi_{--}$is

$$
M_{--}^{2}=g^{2} v^{2}\left(\begin{array}{ccccccc}
2 & -1 & 0 & \cdots & 0 & 0 & 0 \\
-1 & 2 & -1 & \cdots & 0 & 0 & 0 \\
0 & -1 & 2 & \cdots & 0 & 0 & 0 \\
& & & \ddots & & & \\
0 & 0 & 0 & \cdots & -1 & 2 & -1 \\
0 & 0 & 0 & \cdots & 0 & -1 & 2
\end{array}\right) .
$$

Because the field $\phi_{--}$vanishes at the fixed point, we would like to emphasize that the mass spectrum and eigenvector for the physical field are the eigenvalue and eigenvector of above mass matrix. The mass spectrum or eigenvalue is

$$
M_{n}^{2}=4 g^{2} v^{2} \sin ^{2}\left(\frac{(n+1) \pi}{2 N}\right),
$$

where $n=0,1,2, \ldots, N-2$. And the corresponding $n-t h$ eigenvector is

$$
\alpha_{n}=\left(\alpha_{n}^{1}, \alpha_{n}^{2}, \ldots, \alpha_{n}^{N-1}\right),
$$


where

$$
\alpha_{n}^{j}=\frac{\sqrt{2}}{\sqrt{N}} \sin \left(\frac{j(n+1) \pi}{N}\right), j=1,2, \ldots, N-1 .
$$

Moreover, we can discuss the deconstruction of Higgs fields $H_{u}$ and $H_{d}$. We assume that under the $i-t h$ gauge group $S U(5)_{i}$, there is a pair of Higgs 5 -plets $H_{u}^{i}$ and $H_{d}^{i}$ where $i=0,1,2, \ldots, N-1$, and for the $N$-th gauge group, there is a pair of Higgs doublets $H_{u}^{N D}$ and $H_{d}^{N D}$. And we consider the following potential

$$
\begin{aligned}
V= & 2 g^{2} \sum_{i=0}^{N-2}\left(\left|U_{i}\left(H_{u}^{i}\right)^{\dagger}\right|^{2}+\left|H_{u}^{i+1} U_{i}\right|^{2}+\left|U_{i} H_{d}^{i}\right|^{2}+\left|\left(H_{d}^{i+1}\right)^{\dagger} U_{i}\right|^{2}\right) \\
& -\sqrt{2} g^{2} v \sum_{i=0}^{N-2}\left(H_{u}^{i+1} U_{i}\left(H_{u}^{i}\right)^{\dagger}+\left(H_{d}^{i+1}\right)^{\dagger} U_{i} H_{d}^{i}+H . C .\right) \\
& +2 g^{2}\left(\left|U_{w}\left(H_{u}^{(N-1) D}\right)^{\dagger}\right|^{2}+\left|H_{u}^{N D} U_{w}\right|^{2}+\left|U_{w} H_{d}^{(N-1) D}\right|^{2}+\left|\left(H_{d}^{N D}\right)^{\dagger} U_{w}\right|^{2}\right) \\
& -\sqrt{2} g^{2} v\left(H_{u}^{N D} U_{w}\left(H_{u}^{(N-1) D}\right)^{\dagger}+\left(H_{d}^{N D}\right)^{\dagger} U_{w} H_{d}^{(N-1) D}+H . C .\right) .
\end{aligned}
$$

This potential for a pair of Higgs 5-plets $H_{u}^{i}$ and $H_{d}^{i}$ comes from the deconstruction of $\left(D_{5} H_{u}\right)^{\dagger} D_{5} H_{u}$ and $\left(D_{5} H_{d}\right)^{\dagger} D_{5} H_{d}$ in 5-dimensional theory, and the coefficients are determined by the normalization which is compatible with that of gauge fields. So, we obtain that the mass matrix for the doublet $H_{u}^{i D}$ or $H_{d}^{i D}$ is the same as that for the Standard Model gauge boson in Eq. (36), and the mass matrix for the triplet $H_{u}^{i T}$ or $H_{d}^{i T}$ is the same as that for the non-Standard Model gauge boson in Eq. (41). Therefore, similar to the discussions for $A_{\mu}^{a}$ and $A_{\mu}^{\hat{a}}$, the deconstruction results match the continuum results in Table 2 exactly. And then, we solve the doublet-triplet splitting problem.

Furthermore, by counting the number of massless modes, we can prove that the bifundamental fields $U_{i}$ can not match the gauge fields $A_{5}$ because $U_{i} \mathrm{~s}$ are Higgs fields. The correct deconstruction of the $A_{5}$ fields should have 0 massless modes, but, the $U_{i}$ s field will have at least $24 N$ massless modes that are the Goldstone bosons and give masses to the longitudinal components of massive gauge bosons.

In short, the gauge group is broken down to $S U(3) \times S U(2) \times U(1)$ for the zero modes, and the deconstruction results match the continuum results except for $A_{5}$ and $U_{i}$.

\section{Gauge Symmetry Breaking by the Discrete Sym- metry on the Theory Space in the Effective De- construction Scenario}

It seems to us that the Higgs mechanism deconstructions of the gauge symmetry breaking by discrete symmetry on the space-time $M^{4} \times S^{1}$ or $M^{4} \times S^{1} /\left(Z_{2} \times Z_{2}^{\prime}\right)$ 
in the last two sections might not be the real deconstructions. The key point is that, the bifundamental field $U_{i}$, which is the Schwinger line integral along the fifth dimension, should be considered as the field $A_{5}$. However, the mass spectrum and the 5-dimensional wave function for the KK modes of $A_{5}$ can not match those for $U_{i}$ in the Higgs mechanism deconstruction scenario in above two sections by counting the massless modes. In order to have exact match, we propose the effective deconstruction scenario and discuss the gauge symmetry breaking by the discrete symmetry on theory space.

Before we propose the effective deconstruction scenario, let us consider the 5dimensional $S U(5)$ theory with a pair of Higgs 5-plets $H_{u}$ and $H_{d}$ on the space-time $M^{4} \times S^{1}$. If we latticized the fifth dimension with $N+1$ sites, we obtain the following mass terms for the fields $A_{M}^{i}, H_{u}^{i}, H_{d}^{i}$ from the latticized kinetic terms of the fields along the fifth dimension, $i$. e., $\partial_{5} \phi \partial^{5} \phi$ for a generic bulk field $\phi\left(x^{\mu}, y\right)$

$$
\begin{aligned}
V= & \sum_{i=0}^{N-1}\left(\frac{N+1}{2 \pi R}\right)^{2}\left(\frac{1}{2}\left(A_{M}^{i+1}-A_{M}^{i}\right)^{2}+\left|H_{u}^{i+1}-H_{u}^{i}\right|^{2}+\left|H_{d}^{i+1}-H_{d}^{i}\right|^{2}\right) \\
& +\left(\frac{N+1}{2 \pi R}\right)^{2}\left(\frac{1}{2}\left(A_{M}^{N}-\Gamma A_{M}^{0} \Gamma^{-1}\right)^{2}+\left|H_{u}^{N}-\eta_{H_{u}} \Gamma H_{u}^{i}\right|^{2}\right. \\
& \left.+\left|H_{d}^{i+1}-\eta_{H_{d}} \Gamma^{-1} H_{d}^{i}\right|^{2}\right),
\end{aligned}
$$

where the subscript $M$ denotes $0,1,2,3,5$ ( $\mu$ and 5$), \Gamma$ is a $5 \times 5$ matrix and is a generator of $Z_{n}$ group, which is a subgroup of $\pi_{1}\left(S^{1}\right)=Z, i$. e., $\Gamma^{n}=1$. Of course, there exist some other terms, but we are not interested in them here. It is not hard for one to prove that the 5-dimensional $S U(5)$ gauge theory on $M^{4} \times S^{1}$ is equivalent to the 4-dimensional $S U(5)^{N+1}$ gauge theory with above effective potential and other terms in the large $N$ limit, $i$. $e ., N \rightarrow+\infty$. In addition, we would like to point out that the $S U(5)^{N+1}$ gauge theory with above effective potential preserve only the $S U(5) / \Gamma$ gauge symmetry, where the gauge group $S U(5) / \Gamma$ is the commutant of $\Gamma$ in $S U(5)$, mathematically speaking,

$$
S U(5) / \Gamma \equiv\{g \in S U(5) \mid g \Gamma=\Gamma g\} .
$$

Of course, the Lagrangian is not $S U(5)^{N+1}$ gauge invariant, and then, the theory is non-renormalizable. However, this latticized theory is correct because from the point of view of 4-dimensional effective theory, the 5-dimensional $S U(5)$ gauge theory on $M^{4} \times S^{1}$ preserve only the $S U(5) / \Gamma$ gauge symmetry for the zero modes, the gauge symmetries for the non-zero KK modes of 5-dimensional gauge fields are completely broken, and the 5-dimensional theory is non-renormalizable.

Therefore, we can consider the $S U(5)^{N+1}$ gauge theory where in particular the gauge fields $A_{5}$ or the corresponding link fields $U_{i}$ do not have the VEVs, and we introduce above effective potential for the mass terms by hand. In this approach, we can consider the discrete symmetry on theory space and discuss the gauge symmetry breaking. Moreover, the continuum results exactly match the deconstruction results. Because we add the mass terms by hand and our theory is an effective theory, we call 
this deconstruction scenario as effective deconstruction scenario. However, we would like to emphasize that the effective deconstruction scenario is the non-renormalizable theory, and how to construct a renomalizable effective deconstruction scenario deserves further study and is out of the scope of this paper.

\subsection{Deconstruction of $S U(5)$ Breaking on $M^{4} \times S^{1}$ by Wilson Line}

Considering the $S U(5)^{N+1}$ gauge theory with bifundamental link fields $U_{i}$ given in Eq. (7) in subsection 2.2, and assuming that there exists one pair of Higgs 5-plets $H_{u}^{i}$ and $H_{d}^{i}$ under the gauge group $S U(5)_{i}$ where $i=0,1,2, \ldots, N$, we choose the following effective potential

$$
\begin{aligned}
V= & \sum_{i=0}^{N-1}\left(\frac{N+1}{2 \pi R}\right)^{2}\left(\frac{1}{2}\left(A_{\mu}^{i+1}-A_{\mu}^{i}\right)^{2}+\frac{1}{2}\left(U_{i+1}-U_{i}\right)^{2}\right. \\
& \left.+\left|H_{u}^{i+1}-H_{u}^{i}\right|^{2}+\left|H_{d}^{i+1}-H_{d}^{i}\right|^{2}\right) \\
& +\left(\frac{N+1}{2 \pi R}\right)^{2}\left(\frac{1}{2}\left(A_{\mu}^{N}-\Gamma A_{\mu}^{0} \Gamma^{-1}\right)^{2}+\frac{1}{2}\left(U_{N}-\Gamma U_{0} \Gamma^{-1}\right)^{2}\right. \\
& \left.+\left|H_{u}^{N}-\eta_{H_{u}} \Gamma H_{u}^{i}\right|^{2}+\left|H_{d}^{i+1}-\eta_{H_{d}} \Gamma^{-1} H_{d}^{i}\right|^{2}\right)
\end{aligned}
$$

where

$$
\Gamma=\operatorname{diag}(-1,-1,-1,+1,+1)
$$

and $\eta_{H_{u}}=\eta_{H_{d}}=+1$. So, we obtain that the parities of all the fields are the same as those in the subsection 2.1 (see Table 1), and the deconstruction results exactly match the continuum results.

\subsection{Deconstruction of $S U(5)$ Breaking on $M^{4} \times S^{1} /\left(Z_{2} \times Z_{2}^{\prime}\right)$}

We consider the $S U(5)^{4 N}$ gauge theory with bifundamental fields $U_{i}$ as follows ${ }^{4}$

\begin{tabular}{c|cccccc} 
& $S U(5)_{0}$ & $S U(5)_{1}$ & $S U(5)_{2}$ & $\cdots$ & $S U(5)_{N-1}$ & $S U(5)_{N}$ \\
\hline$U_{0}$ & $\square$ & $\square$ & 1 & $\cdots$ & 1 & 1 \\
$U_{1}$ & 1 & $\square$ & $\square$ & $\cdots$ & 1 & 1 \\
$\vdots$ & $\vdots$ & $\vdots$ & $\ddots$ & $\vdots$ & $\vdots$ & \\
$U_{N-1}$ & 1 & 1 & 1 & $\cdots$ & $\square$ & $\square$
\end{tabular}

\footnotetext{
${ }^{4}$ For convenience to define $Z_{2}$ and $Z_{2}^{\prime}$ symmetries, we define half $U_{i}$ as $U_{i}^{\dagger}$ in the previous sections. Because we consider the non-supersymmetric theory, there is no anomaly problem.
} 


\begin{tabular}{c|cccccc} 
& $S U(5)_{N}$ & $S U(5)_{N+1}$ & $S U(5)_{N+2}$ & $\cdots$ & $S U(5)_{2 N-1}$ & $S U(5)_{2 N}$ \\
\hline$U_{N}$ & $\square$ & $\square$ & 1 & $\cdots$ & 1 & 1 \\
$U_{N+1}$ & 1 & $\square$ & $\square$ & $\cdots$ & 1 & 1 \\
$\vdots$ & $\vdots$ & $\vdots$ & $\ddots$ & $\vdots$ & $\vdots$ & \\
$U_{2 N-1}$ & 1 & 1 & 1 & $\cdots$ & $\square$ & $\square$
\end{tabular}.

\begin{tabular}{c|cccccc} 
& $S U(5)_{2 N}$ & $S U(5)_{2 N+1}$ & $S U(5)_{2 N+2}$ & $\cdots$ & $S U(5)_{3 N-1}$ & $S U(5)_{3 N}$ \\
\hline$U_{2 N}$ & $\square$ & $\square$ & 1 & $\cdots$ & 1 & 1 \\
$U_{2 N+1}$ & 1 & $\square$ & $\square$ & $\cdots$ & 1 & 1 \\
$\vdots$ & $\vdots$ & $\vdots$ & $\ddots$ & $\vdots$ & $\vdots$ & \\
$U_{3 N-1}$ & 1 & 1 & 1 & $\cdots$ & $\square$ & $\square$
\end{tabular}

\begin{tabular}{c|cccccc} 
& $S U(5)_{3 N}$ & $S U(5)_{3 N+1}$ & $S U(5)_{3 N+2}$ & $\cdots$ & $S U(5)_{4 N-1}$ & $S U(5)_{0}$ \\
\hline$U_{3 N}$ & $\square$ & $\square$ & 1 & $\cdots$ & 1 & 1 \\
$U_{3 N+1}$ & 1 & $\square$ & $\square$ & $\cdots$ & 1 & 1 \\
$\vdots$ & $\vdots$ & $\vdots$ & $\ddots$ & $\vdots$ & $\vdots$ & \\
$U_{4 N-1}$ & 1 & 1 & 1 & $\cdots$ & $\square$ & $\square$
\end{tabular}.

On the theory space, there are $4 N$ sites and $4 N$ bifundamental link fields. And we would like to add one pair of Higgs 5-plets $H_{u}^{i}$ and $H_{d}^{i}$ on the $i-t h$ site. Moreover, we introduce the following effective potential for the mass terms

$$
\begin{aligned}
V= & \sum_{i=0}^{4 N-1}\left(\frac{2 N}{\pi R}\right)^{2}\left(\frac{1}{2}\left(A_{\mu}^{i+1}-A_{\mu}^{i}\right)^{2}+\frac{1}{2}\left(U_{i}-U_{i+1}\right)^{2}\right. \\
& \left.+\left|H_{u}^{i+1}-H_{u}^{i}\right|^{2}+\left|H_{d}^{i+1}-H_{d}^{i}\right|^{2}\right) .
\end{aligned}
$$

So, the mass matrix for each field is similar to that in Eq. (11).

Now, let us discuss the discrete symmetries on the theory space. We define that $i^{\prime} \equiv i+3 N$ for $0 \leq i<N$ and $i^{\prime} \equiv i-N$ for $N \leq i<4 N$. The $Z_{2}$ and $Z_{2}^{\prime}$ symmetries on theory space are defined by the following equivalent classes

$$
i \sim 4 N-i \text { for } Z_{2}, i^{\prime} \sim 4 N-i^{\prime} \text { for } Z_{2}^{\prime} .
$$


For a generic multiplet $\Phi^{i}\left(x^{\mu}\right)(i=0,1,2, \ldots, 4 N)$ which fills a representation of the gauge group $S U(5)_{i}$, we can define two parity operators $P$ and $P^{\prime}$ for the $Z_{2}$ and $Z_{2}^{\prime}$ symmetries, respectively

$$
\begin{gathered}
\Phi^{i}\left(x^{\mu}\right) \rightarrow \Phi^{4 N-i}\left(x^{\mu}\right)=\eta_{\Phi} P^{l_{\Phi}} \Phi^{i}\left(x^{\mu}\right)\left(P^{-1}\right)^{m_{\Phi}}, \\
\Phi^{i^{\prime}}\left(x^{\mu}\right) \rightarrow \Phi^{4 N-i^{\prime}}\left(x^{\mu}\right)=\eta_{\Phi}^{\prime}\left(P^{\prime}\right)^{l_{\Phi}} \Phi^{i^{\prime}}\left(x^{\mu}\right)\left(P^{\prime-1}\right)^{m_{\Phi}},
\end{gathered}
$$

where $\eta_{\Phi}= \pm 1$ and $\eta_{\Phi}^{\prime}= \pm 1$.

Denoting the physical field $\tilde{\phi}$ with $\left(P, P^{\prime}\right)=( \pm, \pm)$ by $\tilde{\phi}_{ \pm \pm}$, we obtained the physical fields $\tilde{\phi}_{ \pm \pm}^{(n)}\left(x^{\mu}\right)$ expansion in terms of the site fields $\phi^{j}\left(x^{\mu}\right)$

$$
\begin{aligned}
\tilde{\phi}_{++}^{(2 n)}\left(x^{\mu}\right) & =\frac{1}{\sqrt{2 N}} \sum_{j=0}^{4 N-1} \cos \left(\frac{j 2 n \pi}{2 N}\right) \phi^{j}\left(x^{\mu}\right), \\
\tilde{\phi}_{+-}^{(2 n+1)}\left(x^{\mu}\right) & =\frac{1}{\sqrt{2 N}} \sum_{j=0}^{4 N-1} \cos \left(\frac{j(2 n+1) \pi}{2 N}\right) \phi^{j}\left(x^{\mu}\right), \\
\tilde{\phi}_{-+}^{(2 n+1)}\left(x^{\mu}\right) & =\frac{1}{\sqrt{2 N}} \sum_{j=0}^{4 N-1} \sin \left(\frac{j(2 n+1) \pi}{2 N}\right) \phi^{j}\left(x^{\mu}\right), \\
\tilde{\phi}_{--}^{(2 n+2)}\left(x^{\mu}\right) & =\frac{1}{\sqrt{2 N}} \sum_{j=0}^{4 N-1} \sin \left(\frac{j(2 n+2) \pi}{2 N}\right) \phi^{j}\left(x^{\mu}\right),
\end{aligned}
$$

The physical fields $\tilde{\phi}_{++}^{(2 n)}, \tilde{\phi}_{+-}^{(2 n+1)}, \tilde{\phi}_{-+}^{(2 n+1)}$ and $\tilde{\phi}_{--}^{(2 n+2)}$ acquire masses $\frac{4 N}{\pi R} \sin \left(\frac{2 n \pi}{4 N}\right)$, $\frac{4 N}{\pi R} \sin \left(\frac{(2 n+1) \pi}{4 N}\right), \frac{4 N}{\pi R} \sin \left(\frac{(2 n+1) \pi}{4 N}\right)$ and $\frac{4 N}{\pi R} \sin \left(\frac{(2 n+2) \pi}{4 N}\right)$, respectively, where $n=0,1,2$, $\ldots, 2 N-1$. In the large $N$ limit, or $N \rightarrow+\infty$ or $n<<N$, the physical fields $\tilde{\phi}_{++}^{(2 n)}, \tilde{\phi}_{+-}^{(2 n+1)}, \tilde{\phi}_{-+}^{(2 n+1)}$ and $\tilde{\phi}_{--}^{(2 n+2)}$ acquire masses $2 n / R,(2 n+1) / R,(2 n+1) / R$ and $(2 n+2) / R$, respectively. Therefore, the deconstruction results exactly match the continuum results. In addition, zero modes are contained only in $\tilde{\phi}_{++}^{(2 n)}$ fields, thus, the matter content of massless sector is smaller than that of the full multiplets in the theory. Moreover, only $\tilde{\phi}_{++}^{(2 n)}$ and $\tilde{\phi}_{+-}^{(2 n+1)}$ fields have non-zero values at $i=0$ and $i=2 N$, and only $\tilde{\phi}_{++}^{(2 n)}$ and $\tilde{\phi}_{-+}^{(2 n+1)}$ fields have non-zero values at $i=N$ and $i=3 N$.

Under $Z_{2}$ parity $P$, the fields $A_{\mu}^{i}, U_{i}, H_{u}^{i}$ and $H_{d}^{i}$ transform as

$$
\begin{gathered}
A_{\mu}^{4 N-i}\left(x^{\mu}\right)=P A_{\mu}^{i}\left(x^{\mu}\right) P^{-1}, \\
U^{4 N-i}\left(x^{\mu}\right)=-P U^{i}\left(x^{\mu}\right) P^{-1},
\end{gathered}
$$




$$
\begin{gathered}
H_{u}^{4 N-i}\left(x^{\mu}\right)=P H_{u}^{i}\left(x^{\mu}\right), \\
H_{d}^{4 N-i}\left(x^{\mu}\right)=P^{-1} H_{d}^{i}\left(x^{\mu}\right) .
\end{gathered}
$$

And under parity $P^{\prime}$, the gauge field and Higgs field transformations are similar to those under $P$.

We choose the following matrix representations for parity operators $P$ and $P^{\prime}$ that are expressed in the adjoint representation of $S U(5)$

$$
P=\operatorname{diag}(+1,+1,+1,+1,+1), P^{\prime}=\operatorname{diag}(-1,-1,-1,+1,+1),
$$

and then, we obtain that the parities of all the fields are the same as those in the subsection 3.1 (see Table 2), and the deconstruction results exactly match the continuum results.

\section{$5 G^{N}$ Unification}

$G^{N}$ unification has been discussed previously where each gauge group $G$ is broken by the Higgs fields in its adjoint representation [11], or the gauge group $G^{N}$ is broken by introducing more than one link Higgs field between the first two sites recently [6].

The doublet-triplet splitting are also considered in the deconstruction of $S U(5)$ on the disc $D^{2}$ [8]. In this section, we would like to briefly discuss the $G^{N}$ unification where $G^{N}$ is broken down to $S U(3) \times S U(2) \times U(1)^{n-3}$ by the bifundamental link fields in which $n$ is the rank of group $G$. We shall discuss the scenario with $G=S U(5)$ as an example, and similarly, one can discuss the scenario with $G=S U(6), S O(10), E_{6}$, etc, for $\pi_{1}\left(S_{1}\right)=Z[2]$. The discussions for the doublet-triplet splitting are primitive here, and the natural solution to the doublet-triplet splitting problem in a supersymmetric scenario will be presented elsewhere [12].

\section{$5.1 S U(5)_{0} \times S U(5)_{1}$}

The set-up is similar to that in the subsection 2.2 for $N=1$. We choose the VEVs of $U_{0}$ and $U_{1}$ as

$$
\begin{gathered}
<U_{0}>=\operatorname{diag}(v / \sqrt{2}, v / \sqrt{2}, v / \sqrt{2}, v / \sqrt{2}, v / \sqrt{2}) \\
<U_{1}>=\operatorname{diag}(-v / \sqrt{2},-v / \sqrt{2},-v / \sqrt{2}, v / \sqrt{2}, v / \sqrt{2}) .
\end{gathered}
$$

The mass matrix for the Standard Model gauge boson is

$$
M_{S M}^{2}=g^{2} v^{2}\left(\begin{array}{cc}
2 & -2 \\
-2 & 2
\end{array}\right)
$$


and the mass matrix for the non-Standard Model gauge boson is

$$
M_{N S M}^{2}=g^{2} v^{2}\left(\begin{array}{ll}
2 & 0 \\
0 & 2
\end{array}\right) \text {. }
$$

It is easy to check that only the Standard Model gauge bosons have zero modes and the non-Standard Model gauge bosons are massive.

Now, let us discuss the doublet-triplet splitting. Suppose that $H_{u}^{i}$ and $H_{d}^{i}$ are in the fundamental and anti-fundamental representations of $S U(5)_{i}$ respectively, where $i=0,1$. With the following potential

$$
\begin{aligned}
V= & 2 g^{2}\left(\left|U_{0}\left(H_{u}^{0}\right)^{\dagger}\right|^{2}+\left|H_{u}^{1} U_{0}\right|^{2}+\left|U_{0} H_{d}^{0}\right|^{2}+\left|\left(H_{d}^{1}\right)^{\dagger} U_{0}\right|^{2}\right) \\
& +2 g^{2}\left(\left|U_{1}\left(H_{u}^{1}\right)^{\dagger}\right|^{2}+\left|H_{u}^{0} U_{1}\right|^{2}+\left|U_{1} H_{d}^{1}\right|^{2}+\left|\left(H_{d}^{0}\right)^{\dagger} U_{1}\right|^{2}\right) \\
& -\sqrt{2} g^{2} v\left(H_{u}^{1} U_{0}\left(H_{u}^{0}\right)^{\dagger}+\left(H_{d}^{1}\right)^{\dagger} U_{0} H_{d}^{0}+\text { H.C. }\right) \\
& -\sqrt{2} g^{2} v\left(H_{u}^{0} U_{1}\left(H_{u}^{1}\right)^{\dagger}+\left(H_{d}^{0}\right)^{\dagger} U_{1} H_{d}^{1}+\text { H.C. }\right)
\end{aligned}
$$

we obtain that the mass matrix for the doublet $H_{u}^{i D}$ or $H_{d}^{i D}$ is the same as that for the Standard Model gauge boson in Eq. (78), and the mass matrix for the triplet $H_{u}^{i T}$ or $H_{d}^{i T}$ is the same as that for the non-Standard Model gauge boson in Eq. (79). Therefore, we solve the doublet-triplet splitting problem.

The possible interesting model is that we put three 5 -plets $(\overline{5})$ fermions under $S U(5)_{0}$, and three 10-plets $(10)$ fermions under $S U(5)_{1}$. Then, the proton decay is suppressed at least at one loop level. Of course, there might exist anomaly in the model. One way to avoid anomaly is that, we consider supersymmetry and three link fields that are in the fundamental and anti-fundamental representations of $S U(5)_{0}$ and $S U(5)_{1}$, respectively.

\section{$5.2 S U(5)_{0} \times S U(5)_{1} \times S U(5)_{2}$}

The set-up is similar to that in the subsection 2.2 for $N=2$. However, we choose the following VEVs of $U_{0}, U_{1}$ and $U_{2}$, which can also give the correct mass matrices for gauge boson and Higgs fields

$$
<U_{i}>=\operatorname{diag}(-v / \sqrt{2},-v / \sqrt{2},-v / \sqrt{2}, v / \sqrt{2}, v / \sqrt{2}), \text { for } i=0,1,2 .
$$

The mass matrix for the Standard Model gauge boson is

$$
M_{S M}^{2}=g^{2} v^{2}\left(\begin{array}{ccc}
2 & -1 & -1 \\
-1 & 2 & -1 \\
-1 & -1 & 2
\end{array}\right)
$$


and the mass matrix for the non-Standard Model gauge boson is

$$
M_{N S M}^{2}=g^{2} v^{2}\left(\begin{array}{ccc}
2 & +1 & +1 \\
+1 & 2 & +1 \\
+1 & +1 & 2
\end{array}\right) \text {. }
$$

It is easy to check that only the Standard Model gauge bosons have zero modes and the non-Standard Model gauge bosons are massive.

Now, let us discuss the doublet-triplet splitting. Suppose that $H_{u}^{i}$ and $H_{d}^{i}$ are in the fundamental and anti-fundamental representations of $S U(5)_{i}$ respectively, where $i=0,1,2$. With the following potential

$$
\begin{aligned}
V= & 2 g^{2}\left(\left|U_{0}\left(H_{u}^{0}\right)^{\dagger}\right|^{2}+\left|H_{u}^{1} U_{0}\right|^{2}+\left|U_{0} H_{d}^{0}\right|^{2}+\left|\left(H_{d}^{1}\right)^{\dagger} U_{0}\right|^{2}\right) \\
& 2 g^{2}\left(\left|U_{1}\left(H_{u}^{1}\right)^{\dagger}\right|^{2}+\left|H_{u}^{2} U_{1}\right|^{2}+\left|U_{1} H_{d}^{1}\right|^{2}+\left|\left(H_{d}^{2}\right)^{\dagger} U_{1}\right|^{2}\right) \\
& +2 g^{2}\left(\left|U_{2}\left(H_{u}^{2}\right)^{\dagger}\right|^{2}+\left|H_{u}^{0} U_{2}\right|^{2}+\left|U_{2} H_{d}^{2}\right|^{2}+\left|\left(H_{d}^{0}\right)^{\dagger} U_{2}\right|^{2}\right) \\
& -\sqrt{2} g^{2} v\left(H_{u}^{1} U_{0}\left(H_{u}^{0}\right)^{\dagger}+H_{u}^{2} U_{1}\left(H_{u}^{1}\right)^{\dagger}+H_{u}^{0} U_{2}\left(H_{u}^{2}\right)^{\dagger}+\text { H.C. }\right) \\
& -\sqrt{2} g^{2} v\left(\left(H_{d}^{1}\right)^{\dagger} U_{0} H_{d}^{0}+\left(H_{d}^{2}\right)^{\dagger} U_{1} H_{d}^{1}+\left(H_{d}^{0}\right)^{\dagger} U_{2} H_{d}^{2}+\text { H.C. }\right),
\end{aligned}
$$

we obtain that the mass matrix for the doublet $H_{u}^{i D}$ or $H_{d}^{i D}$ is the same as that for the Standard Model gauge boson in Eq. (82), and the mass matrix for the triplet $H_{u}^{i T}$ or $H_{d}^{i T}$ is the same as that for the non-Standard Model gauge boson in Eq. (83). Thus, we solve the doublet-triplet splitting problem.

The possible interesting model is that we put one family $(\overline{5}+10)$ fermions under one $S U(5)$ gauge group, and define the $Z_{3}$ symmetry on the model.

\section{Discussion and Conclusion}

An interesting question is how to realize the Yukawa couplings in the deconstruction scenarios. If the 5-dimensional theory is non-supersymmetric, we can consider the 5-dimensional Yukawa couplings or 3-brane localized Yukawa couplings. In the deconstruction scenarios, we need to introduce the Yukawa couplings on all the sites for the first case. For the second case, we introduce the Yukawa couplings only on the corresponding particular site for that 3-brane. For example, if the 3-brane localized Yukawa couplings is on the 3-brane at $y=0$ in the 5-dimensional theory, we only introduce the Yukawa couplings on the $0-t h$ site. In addition, if the 5-dimensional theory is supersymmetric, we can only consider the Yukawa couplings (superpotential) on the observable 3 -brane at the fixed point because the 5 -dimensional $N=1$ supersymmetry is 4-dimensional $N=2$ supersymmetry, and then, the bulk Yukawa couplings are forbidden. In the deconstruction scenarios, we introduce the Yukawa couplings (superpotential) on the corresponding site for the observable 3-brane at the fixed point. 
In this paper, we deconstruct the non-supersymmetric $S U(5)$ breaking by discrete symmetry on the space-time $M^{4} \times S^{1}$ and $M^{4} \times S^{1} /\left(Z_{2} \times Z_{2}^{\prime}\right)$ in the Higgs mechanism deconstruction scenario. And we explain the subtle point on how to exactly match the continuum results with the latticized results on the quotient space $S^{1} / Z_{2}$ and $S^{1} /\left(Z_{2} \times Z_{2}^{\prime}\right)$. Because it seems to us that the Higgs mechanism deconstruction scenario might not be the real deconstruction, we propose the effective deconstruction scenario and discuss the gauge symmetry breaking by the discrete symmetry on theory space in this approach. However, for simplicity, we do not consider supersymmetry and only discuss GUT group $S U(5)$. So, it is interesting to consider supersymmetry and discuss other GUT breaking, for instance, $S U(6), S O(10)$, and $E_{6}$, etc. Moreover, we can study the deconstruction of the extra space manifolds like the two-torus $T^{2}$, the disc $D^{2}$ and the annulus $A^{2}$, and discuss the general gauge symmetry and supersymmetry breaking by the discrete symmetry on the general theory space in the effective deconstruction scenario. It seems to us that we will have the link fields and Higgs unification, suppress the proton decay by R-symmetry, solve the doublet-triplet splitting problem and $\mu$ problem.

As an application, we suggest the $G^{N}$ unification where $G^{N}$ is broken down to $S U(3) \times S U(2) \times U(1)^{n-3}$ by the bifundamental link fields and the doublet-triplet splitting can be achieved. Furthermore, we can consider the general link fields, for example, $(10, \overline{10})$ for $S U(5)_{0} \times S U(5)_{1}$. In short, with the general $G^{N}$ unification, we wish we can solve the tough problems in the traditional 4-dimensional GUT models.

\section{Acknowledgments}

We would like to thank W. Liao for helpful discussions. This research was supported in part by the U.S. Department of Energy under Grant No. DOE-EY-76-02-3071.

\section{References}

[1] Y. Kawamura, Prog. Theor. Phys. 103 (2000) 613 hep-ph/9902423, hepph/0012125, hep-ph/0012352; G. Altarelli and F. Feruglio, hep-ph/0102301; L. Hall and Y. Nomura, hep-ph/0103125; T. Kawamoto and Y. Kawamura, hep-ph/0106163; A. Hebecker and J. March-Russell, hep-ph/0106166, hepph/0107039; R. Barbieri, L. Hall and Y. Nomura, hep-ph/0106190, hepth/0107004; A. B. Kobakhidze, hep-ph/0102323; T. Li, hep-th/0107136, hepph/0108120; J. A. Bagger, F. Feruglio and F. Zwirner, hep-th/0107128; A. Masiero, C. A. Scrucca, M. Serone and L. Silvestrini, hep-ph/0107201; L. Hall, H. Murayama and Y. Nomura, hep-th/0107245; C. Csaki, G. D. Kribs and J. Terning, hep-ph/0107266; L. Hall, Y. Nomura and D. Smith, hep-ph/0107331; N. Maru, hep-ph/0108002; T. Asaka, W. Buchmuller and L. Covi, hepph/0108021; L. Hall, Y. Nomura, T. Okui and D. Smith, hep-ph/0108071; N. Haba, T. Kondo, Y. Shimizu, T. Suzuki and K. Ukai, hep-ph/0108003; 
L. Hall, J. March-Russell, T. Okui and D. Smith, hep-ph/0108161; R. Dermisek and A. Mafi, hep-ph/0108139; T. Watari and T. Yanagida, hep-ph/0108152; Y. Nomura, hep-ph/0108170; Q. Shafi and Z. Tavartkiladze, hep-ph/0108247; L. Hall, J. March-Russell, T. Okui and D. Smith, hep-ph/0108161; N. Haba, hep-ph/0110164; R. Barbieri, L. Hall and Y. Nomura, hep-ph/0110102; L. Hall and Y. Nomura, hep-ph/0111068; M. Kubo, C. S. Lim, H. Yamashita, hepph/0111327; G. Bhattacharyya and K. Sridhar, hep-ph/0111345; H. D. Kim, J. E. Kim, H. M. Lee, hep-ph/0112094; N. Haba, T. Kondo, Y. Shimizu, hepph/0112132; A. Hebecker, hep-ph/0112230; N. Haba, T. Kondo, Y. Shimizu, hep-ph/0202191; T. Li and W. Liao, hep-ph/0202090; L. J. Hall and Y. Nomura, hep-ph/0202107; S. Dimopoulos, D. E. Kaplan and N. Weiner, hep-ph/0202136; K. W. Choi, H. D. Kim and Y. W. Kim, hep-ph/0202257; A. Hebecker and J. March-Russell, hep-ph/0204109.

[2] T. Li, hep-th/0110065; hep-th/0112255; C. S. Huang, J. Jiang, T. Li and W. Liao, hep-th/0112046.

[3] N. Arkani-Hamed, A. G. Cohen and H. Georgi, hep-th/0104005; C. T. Hill, S. Pokorski and J. Wang, hep-th/0104035; M. J. Strassler, hep-th/0104032.

[4] N. Arkani-Hamed, A. G. Cohen and H. Georgi, hep-ph/0105239; hep-th/0108089; H. Cheng, C. T. Hill, S. Pokorski and J. Wang, hep-ph/0104179; H. Cheng, C. T. Hill and J. Wang, hep-ph/0105323; C. Csaki, J. Erlich, C. Grojean and G. D. Kribs, hep-ph/0106044; H. C. Cheng, D. E. Kaplan, M. Schmaltz and W. Skiba, hep-ph/0106098; P. H. Chankowski, A. Falkowski and S. Pokorski, hep-ph/0109272; N. Arkani-Hamed, A. G. Cohen, D. B. Kaplan, A. Karch and L. Motl, hep-th/0110146; I. Rothstein and W. Skiba, hep-th/0109175; M. Alishahiha, hep-th/0105153; A. Adams and M. Fabinger, hep-th/0111079; K. Sfetsos, hep-th/0106126; C. T. Hill, hep-th/0109068; C. Csaki, J. Erlich, V. V. Khoze, E. Poppitz, Y. Shadmi and Y. Shirman, hep-th/0110188; M. Bander, hep-th/0107130; W. Skiba and D. Smith, hep-ph/0201056; Z. Berezhiani, A. Gorsky and I. I. Kogan, hep-th/0203016; R. Sekhar Chivukula and HongJian He, hep-ph/0201164; N. Arkani-Hamed, A. G. Cohen, T. Gregoire and J. G. Wacker, hep-ph/0202089; K. Lane, hep-ph/0202093; A. Falkowski, C. Grojean and Stefan Pokorski, hep-ph/0203033; Z. Chacko, E. Katz and E. Perazzi, hep-ph/0203080; T. Kobayashi, N. Maru and K. Yoshioka, hep-ph/0110117.

[5] C. Csaki, G. D. Kribs and J. Terning, hep-ph/0107266.

[6] H. Cheng, K. T. Matchev and J. Wang, hep-ph/0107268.

[7] N. Weiner, hep-ph/0106097.

[8] E. Witten, hep-ph/0201018.

[9] M. B. Halpern and W. Siegel, Phys. Rev. D11 (1975) 2967.

[10] T. Li, Phys. Lett. B 478 (2000) 307; Nucl. Phys. B 582 (2000) 176. 
[11] A. Davidson and K. C. Wali, Phys. Rev. Lett. 58 (1987) 2623; P. Cho, Phys. Rev. D 48 (1993) 5331, R. N. Mohapatra, Phys. Lett. B 379 (1996) 115.

[12] T. Li and W. Liao, in preparation. 